Journal of Computer Science 8 (1): 163-169, 2012

ISSN 1549-3636

(C) 2012 Science Publications

\title{
An Efficient Hand-off Mechanism for Vehicular Networks
}

\author{
${ }^{1} \mathrm{M}$. Dinakaran and ${ }^{2} \mathrm{P}$. Balasubramanie \\ ${ }^{1}$ School of IT and Engineering, VIT University, South India \\ ${ }^{2}$ School of Computer Technology and Applications, \\ Kongu Engineering College, South India
}

\begin{abstract}
Problem statement: In recent times, wireless networks have become ubiquitous and the hotspot research arena. When a mobile node switches from one access network to another network, the node is said to perform a handoff. This transition is a complex and time-consuming task since after transit the mobile node has to obtain a new Care of Address (CoA) from the transited AR and bind this CoA with its home agent, apart from this there occurs a problem of packet loss. However there exist some solutions for this handoff problem, these mechanisms do not offer efficient solution for the problem. Approach: A mechanism to reduce the hand off delay in vehicular networks is proposed in this article. This approach pre allocates the new address for the mobile node before hand off based on its mobility. Results: The proposed system is implemented in ns 2 simulation environment and compared with some existing hand of mechanisms and its evident that the proposed mechanism reduces the hand off delay and packet loss ratio. Conclusion: This article is concerned with defining a better solution for the handoff problem such that the proposed mechanism offers efficient results compared to the existing system.
\end{abstract}

Keywords: Hand off, mobile networks, NEMO hand off, Internet Engineering Task Force (IETF), Care of Address (CoA), Network Mobility (NEMO), Binding Update (BU), Binding Acknowledgement (BA)

\section{INTRODUCTION}

Mobile phones are very essential device for business and personal usages for all now a days (Nasir et al., 2008). IP is the Internet Protocol developed by Internet Engineering Task Force (IETF). When a node changes its point of attachment, this protocol informs the network about the details regarding the switching of host to a new access network (QS, 2010). It provides location-independent access to Internet. Each mobile host is assigned a home address when it is present inside the home network. When the mobile host moves out of the home network, it is identified by the Care of Address $(\mathrm{CoA})$ which is registered with the home agent (Abdelgadir et al., 2011). Mobile IP specifies the procedure of how a mobile node registers its CoA with the home agent and how the home agent routes the packets to the mobile node (Beijnum, 2006). Mobile IP is mostly used in wireless networks where mobile devices traverses across multiple LANs (Hassan and Hassan, 2011).To support the movement of a complete network which changes its point of attachment to the fixed infrastructure and to maintain the sessions of the mobile nodes uninterrupted, the concept of Network Mobility (NEMO) was proposed. To support this network mobility, the mobile network must contain at least a single router which maintains the contact with the fixed infrastructure and maintains the sessions of individual hosts inside the network.

In NEMO every mobile router is given two addresses namely the permanent home address given by the home agent and the care of address given by the access networks that the mobile router visits (Beijnum, 2006). When the mobile node moves out of the home network, it sends a request for a care of address from the new access router. After receiving the new CoA, mobile router has to register this $\mathrm{CoA}$ to the home agent (Bernardos et al., 2005). So, MR sends a Binding Update (BU) to HA informing about its new CoA and also sends the prefixes of mobile nodes within the network so that the HA can route the datagram addressed for the MNN. The home agent after receiving BU replies MR with a Binding Acknowledgement (BA) (Devarapalli et al., 2005). A positive acknowledgement insists that the home agent is ready to forward the datagram meant for MNN to MR.

After the binding process is successfully completed, a bi-directional tunnel is established between the HA and the MR with one end point as Home Agent's address and the other end point being the MR's Care of Address (Devarapalli et al., 2005). Once the bi-directional tunnel is established Corresponding node can send message to MNN and vice versa. When a corresponding node wants 
to send a datagram to a $\mathrm{MNN}$, the datagram is first transmitted to the home network of MNN (Devarapalli et al., 2005). In home network, HA encapsulates the packet with source address being its home address and destination address as the MR's CoA. This packet is then transmitted to MR's CoA. At this point, MR removes the outer IPV6 header from the encapsulated packet and delivers the datagram to the respective MNN. Before decapsulating the tunneled packet, MR must make sure that the source address on outer IPV6 header is Home Agent's address. It should also check if the destination address in innerIPV6 belongs to the prefix of the mobile network. This type of packet transfer ensures transparency between the nodes as either the $\mathrm{CN}$ or the $\mathrm{MNN}$ is aware of the packet encapsulation and decapsulation.

Literature survey: HANF-OFF: With the increased demand of user mobility, wireless networks have become ubiquitous. The major problem concerning wireless networks is the handoff. Handoff occurs when a mobile node $\mathrm{MN}$ or mobile router MR moves from one coverage area to another. When it moves to a new coverage area, MR has to register with the new AR and continue internet access. There occurs time delay in this process and is considered as bottleneck condition for wireless networks. When a mobile router moves from one access network to another, MR has to register with the new network and retain its existing sessions. This switching from one network to another is called as Handoff. Handoff latency occurs due to two reasons namely registration delay and binding latency. Registration latency is the time taken by the MR to request and obtain the care of Address from new access network. This consists of movement detection latency, the time taken to detect the movement of mobile router and CoA configuration latency i.e., the time taken for MR to request CoA from new network and time taken for new CoA allotment (Sharma et al., 2004). Binding latency comprises of delay time taken by MR to send the binding update to home agent and the time taken to receive binding acknowledgement from HA.

This delay often occurs because when MR moves from one network to another, the following has to take place (Johnson, 2003):

- New network has to check the authentication of the mobile router's identity

- Undo registration at old network and register at new network

- De-allocate resources at older network and allocate in newer network

- Update network registry about the switching to another network

- Configure CoA from network and bind
This handoff is classified into two types namely Micro and Macro mobility. When the mobile router switches to a network within a single administrative domain, it is known as micro mobility or intra-domain handoff. Micro mobility doesn't cause much delay and are easy to resolve. When mobility takes place over a large region comprising of various networks under different administrative domains, it is called as Macro mobility or Inter-domain handoff. However macro mobility possesses the problem of overhead, path reestablishment delay and packet loss (Johnson, 2003). The objective of the protocol is to offer reliable and uninterrupted Internet services independent of the mobility and location of the router yet maintaining all the sessions of the mobile network nodes enabling undisturbed service either across a single domain or across several domains.

Hierarchical handoff mechanism: To manage the global (macro) mobility and local (micro) mobility independently, the Hierarchical architecture is proposed. Mobile IPv6 protocol doesn't support any kind of hierarchy. Both micro and macro mobility is considered the same. In Mobile IP, a Binding Update is sent to correspondent node and home agent whenever a mobile node changes its point-of-attachment to the Internet. Therefore the signaling load is the same independent of the mobility pattern. The hierarchical protocol separates the local mobility (intra-site) and the global mobility (inter-site) of the mobile nodes (Castelluccia, 1998). Local handoffs are managed locally and transparently. The intra-site mobility is not visible to the correspondent node. Hierarchical architecture introduces the concept of domain or site. A domain is an administrative arena where many access routers are under the administrative control of a single router called MAP i.e., Mobile Anchor Point (Zulkeflee, 2011). Every domain consists of at least single MAP and in case of multiple hierarchies consists of many MAPs. The IP address of MAP is regarded as Regional Care of Address ( $\mathrm{RCoA}$ ). The address given to the mobile router or mobile node is regarded as Local Care of Address (LCoA). The simplest hierarchical architecture consists of single MAP however in complex architectures multiple MAPs are present. However the IP address of lowest architecture MAP is considered as RCoA.

Fast handoff mechanism: The Fast Handoff mechanism is used to configure a new address to the mobile host before it performs the actual transition from one place to the other (Sulander et al., 2005). This mechanism is used to reduce the latency period. It consists of three phases: handover initiation, tunnel establishment and packet forwarding (Huang, 2006). 
The first and foremost complexity is that the access routers have to detect the movement of MR using any movement detection algorithm and it adds to the load of AR. Fast handoff makes use of new messages like Proxy router requests and advertisements. Hence new standards apart from existing have to be adopted. Though Fast handoff contributes to time delay reduction it does not proposes any methodology for packet losses and packet re-ordering.

Proposed hand-off mechanism: The proposed system named as incessant hand off mechanism facilitates the combination of advantages of hierarchical and fast handoff mechanisms. The system operates in hierarchical environment and applies the principles of fast handoff mechanism. This proposed model eliminates the disadvantages posed by both the existing models and solves the problem of packet loss also. The incessant hand off system is based on the hierarchical architecture and hence any change in the architectural level is not needed. Further the system does not demand any change in the mobile router or mobile node and hence greatly flexible. However the system demands change in the software level configuration of the MAPs and Access Routers. This change does not impose any difficulty over the functionality of the system and increases the efficiency to a larger extent. The system demands the MAP to send pre-allocated tables before handoff and consumes a little memory space. Figure 1 shows the incessant hand off architecture.

Thus the implementation of the proposal doesn't need any huge changes but increases the communication efficiency. Implementation of the proposal requires the architecture as that of hierarchical architecture. Here, Mobile Anchor Point (MAP) takes the entire responsibility of managing the access routers within its domain i.e., MAP takes care of routing of packets and allocation of CoA. Each MR is allocated two addresses namely Regional Care of Address (RCoA) and onLink Care of Address (LCoA). RCoA represents the address of MAP responsible for the domain and LCoA represents the CoA allotted for the MR. When MAP detects the movement of MR, it assumes that MR is about to perform a handoff. But it is unaware of either the handoff is intra-domain or interdomain. For every access router within the domain, MAP pre-allocates LCoA for moving MR. Information regarding this pre-allocated $\mathrm{LCoA}$ in corresponding access routers is stored in the form of table known as intra-domain pre-allocated address table. This table is sent to all routers within the domain by limited broadcast routing. Since MAP is unaware of the type of handoff, it also sends intimation message containing LCoA and RCoA of the moving MR to neighborhood
MAPs. Here both LCoA and RCoA are being included to identify the individual node when more than one node performs handoff simultaneously. These neighborhood MAPs create Inter-domain Pre-allocated Address table where reserved LCoA for the moving MR in various access routers are stored. The following are the sample tables for intra and inter domain pre allocated address.

Intra-domain Pre-allocated address table is created by MAP under whose range is MR present. This table contains the present LCoA of moving MR present in the first column. The second column contains the access router's identification. This denotes the router IP address. The third column contains nLCoA that is the addressed reserved for moving MR under each access router. A sample table information is upadated in Table 1. As a row the table indicates the router address and the address reserved for MR under the specified router. This table is distributed to all routers within domain using limited broadcast routing. The access routers on receiving the packet, matches the table with its IP address. The row which matches the router IP address on the second column is chosen and stored separately in the buffer. Thus the load on the AR is reduced. Only small storage is needed even if multiple handoffs take place simultaneously.

Inter-domain pre-allocated address table is created by neighborhood MAPs once they are intimated about the moving MR. This table is being used when interdomain handoff occurs and hence the name. This table contains the present LCoA and RCoA of the moving MR in its first and second column. RCoA indicates the present MAP address. Present LCoA is being added in the table to uniquely identify the individual MR. This is because there is a supposition that more than one MR present under the same RCoA performs handoff. The third column indicates the access router identification and the forth column contains the nLCoA, the reserved address for moving MR under the access router specified in the third column. A sample table information is upadated in Table 2. This table is distributed to all access routers within the domain using limited broadcast routing. The ARs on obtaining the table checks for the row matching with its IP address.

Table 1: Intra-domain pre-allocated address Table

\begin{tabular}{lll}
\hline LCoA & Router address & nLCoA \\
\hline 101 & AR1 & 102 \\
101 & AR2 & 103 \\
\hline
\end{tabular}

Table 2: Inter-domain pre-allocated address Table
\begin{tabular}{llll}
\hline RCoA & LCoA & Router address & nLCoA \\
\hline 0416 & 101 & AR3 & 104 \\
0416 & 101 & AR5 & 105 \\
\hline
\end{tabular}




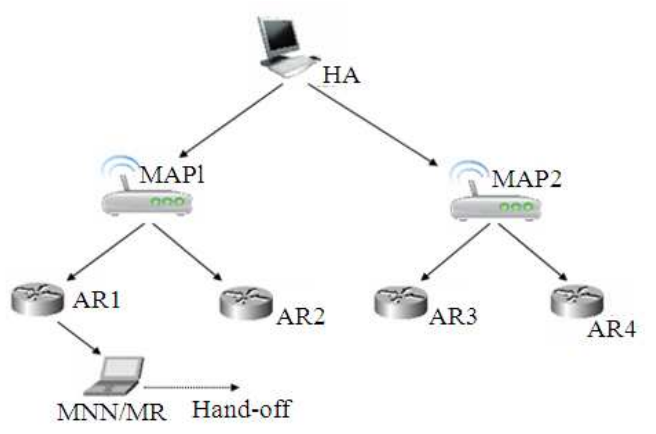

Fig. 1: Incessant hand-off mechanism

On successful search, it stores the particular row in its buffer and ignores the rest. Hereby the load on the AR memory space is lowered and even in case of simultaneous multiple handoffs only small memory space is demanded. This buffer space is however refreshed after specified period. The buffer is refreshed for every $3 \mathrm{~min}$. When a condition of buffer overflow occurs, the early entries in the table are deleted. Thus the tables are maintained in the AR buffers and demand no extra effort.

Intra-domain handoff: Intra-domain handoff occurs when the movement of the mobile router takes place within a single administrative domain or site. This is also called as micro-mobility. Since the proposed mechanism operates in the same environment as that of hierarchical architecture, there is no much difference in the handoff procedure except that the address is already reserved for the moving MR. Once the mobile router starts moving, MAP identifies the movement of MR using any one of the movement detection algorithm. Once the movement is detected, the MAP creates a intra-domain pre-allocated address table reserving the address for the moving MR in each and every router within its administration and intimates the nearby MAPs regarding the LCoA and RCoA of the moving MR. The nearby MAPs create Inter-domain preallocated address table reserving the address for the moving MR. Once MR performs an intra-domain handoff that is it moves to the range of a new access router within the same administrative domain of the previous access router, the new access router searches the reserved table to verify if there is an address reserved for the MR that's has come into its range. If the new access router finds an entry for the MR, it simply allocates the address specified in the table and sends a registration update to its immediate upper MAP. MAP updates its binding cache and acts as local home agent. In case of multiple hierarchies of MAPs, this MAP intimates the registration update to all its nearby MAPs and its upper administrative MAP. After intimation, MAP acts as the local home agent and receives all packets destined to MR. However intradomain transition is not intimated to the home agent and correspondent nodes and so the burden of sending Binding Update and receiving Binding Acknowledgement is greatly reduced. Here the procedure of MR sending a local binding update to MAP and MAP sending local binding acknowledgement is also reduced as the AR takes up the responsibility of informing the update to MAP. Thus the registration delay for intra-domain incessant handoff mechanism is greatly reduced. Due to decreased delay time, the packet drop is reduced.

Inter-Domain Handoff: When the movement of the mobile router takes place from one administrative domain to another administrative domain, it is called as inter-domain or macro-mobility. This is a longer process than intra-domain since it is outside the domain; the movement has to be informed to all the home agent and correspondent nodes. Let us consider that a mobile router lies under a site. When the mobile router starts moving, MAP in the site detects the movement of MR and performs the preliminary actions. Preliminary actions include creating an intra-domain pre-allocated address table by the MAP. This table contains the LCoA of the moving MR. This table assigns a new LCoA for moving MR under each access router within its domain. However the existing AR taking care of moving MR is not allocated any new address. This table contains the address of each access router and LCoA that is assigned under these access routers for the moving MR. However the MAP is unaware if MR performs only intra-domain handoff. So the existing MAP indicates the movement of MR to nearby MAPs by informing the present LCoA and RCoA of the MR. LCoA is included to identify the unique MR as there is a possibility that more than one MR performing handoff. Once the neighborhood MAPs receives the information, these MAPs create interdomain pre-allocation address table. This table reserves the address for moving MR under each of the access routers in neighborhood MAPs. This table is distributed to all routers using limited broadcast. These reservations are made even before the actual handoff takes place and is called as preliminaries. When interdomain handoff takes place, MN moves from one 
domain to another. MR sends neighborhood acknowledgement to the access router in its vicinity. The new access router on receiving NA, checks its buffer if there is an address reserved for the MR. In case of successful search, new AR allocates the address reserved for it and intimates the registration update to its immediate upper MAP. In case of multiple hierarchies the updating occurs in same way as that of intra-domain. Inter-domain transition has to be intimated to the HA and so MAP sends binding update to the HA of MR. HA authenticates the BU and on successful authentication, responds with Binding Acknowledgement. After these transactions, a bidirectional tunnel is formed between the HA and new MAP and all packets are forwarded through the tunnel. Thus inter-domain handoff registration is successfully registered and packet flow takes place uninterrupted.

\section{MATERIALS AND METHODS}

We present an analysis of the three handoff mechanisms namely hierarchical, fast and incessant handoff mechanism based on the registration time delay. When MR starts moving away from the range of an AR, the control has to be transferred to nearby AR to support continuous internet access. There occur several processes before the switching occurs and the time taken for these processes refer to registration delay in the network. The overall handoff mechanism is being analyzed by two factors namely

Registration delay: Registration delay indicates the time taken by mobile router or mobile node to register itself to a new access router and have a continuous internet access router after it has performed handoff. Lower the registration delay, higher is the efficiency of the system.

Packet loss: Packet loss defines the number of packets dropped for a defined number of packet transactions. Lower the packet drop, higher is the efficiency of the system.

\section{RESULTS}

The following Fig. 2 graph shows that the hand off registration delay of fast hand off mechanism. Figure 3 graph shows the registration delay of hierarchical hand off mechanism. Figure 4 graph shows the registration delay of proposed Incessant hand off mechanism.

The following Fig. 5 graph shows that the packets dropped in fast hand off mechanism. Figure 6 graph shows the packets dropped in hierarchical hand off mechanism. Figure 7 graph shows the packets dropped in proposed Incessant hand off mechanism.

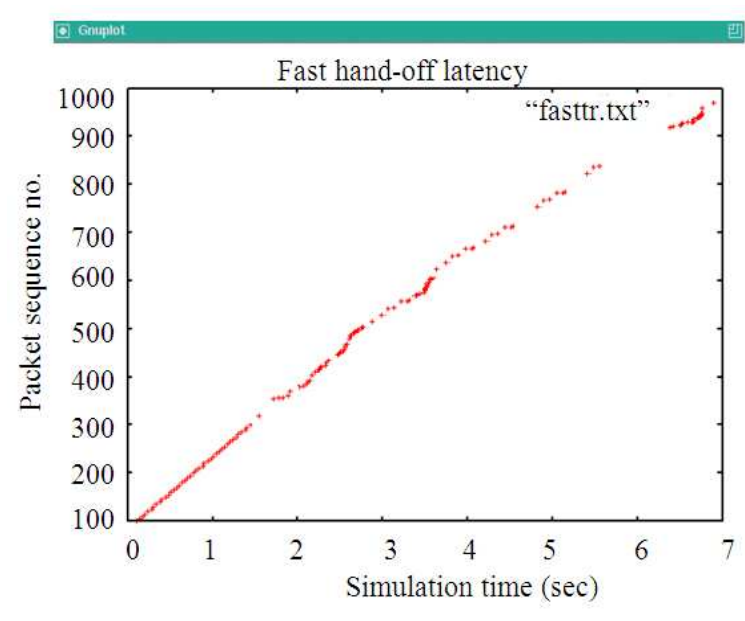

Fig. 2: Fast handoff registration delay

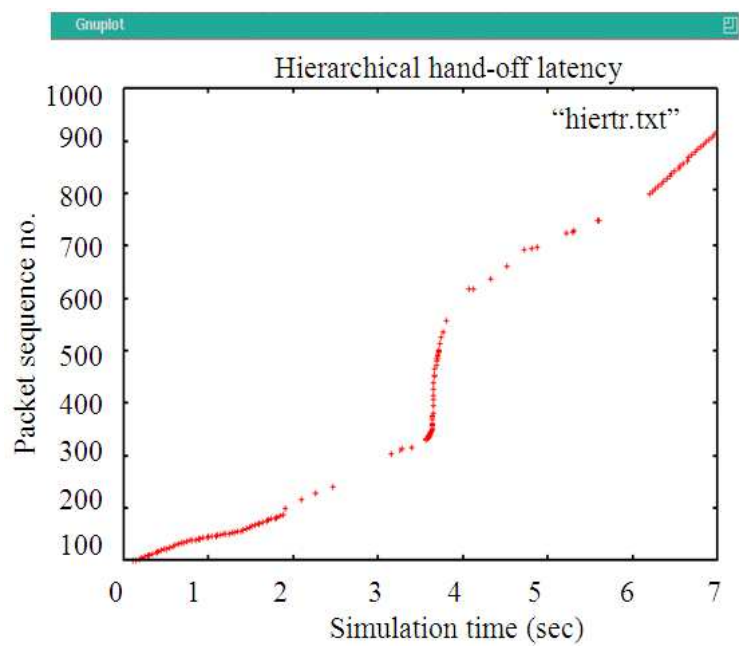

Fig. 3: Hierarchical handoff registration delay

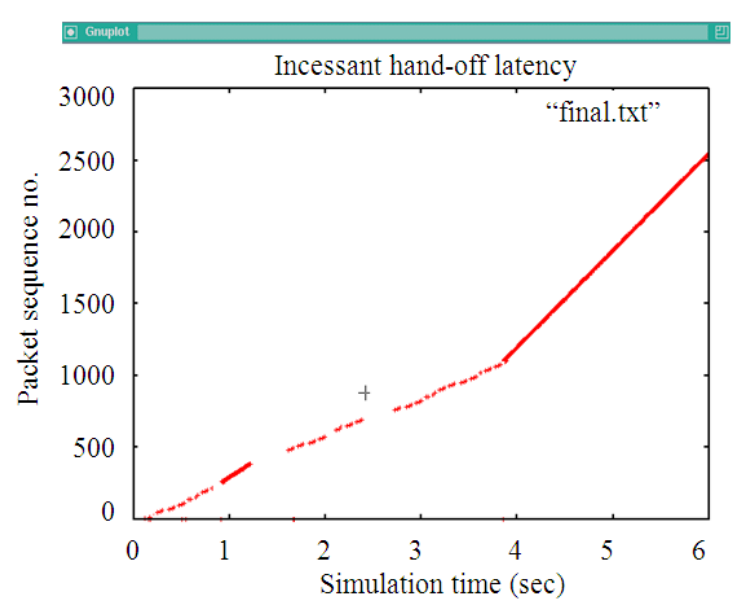

Fig. 4: Incessant handoff registration delay 


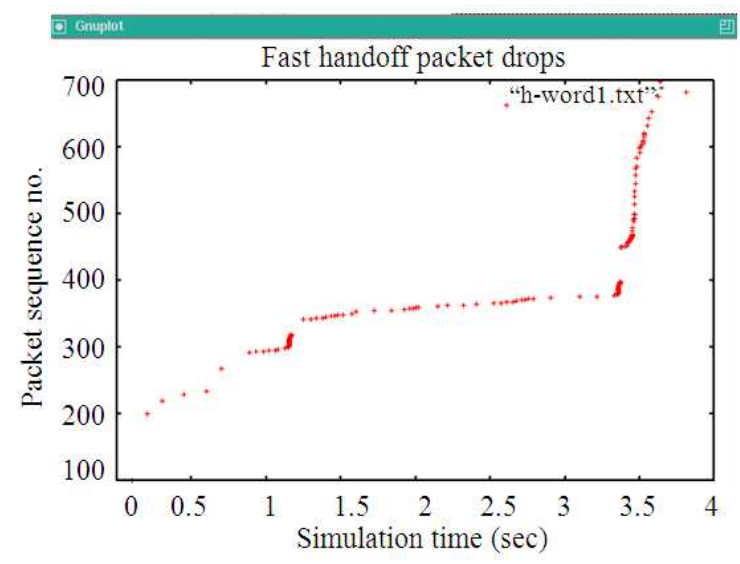

Fig. 5: Packet loss fast handoff

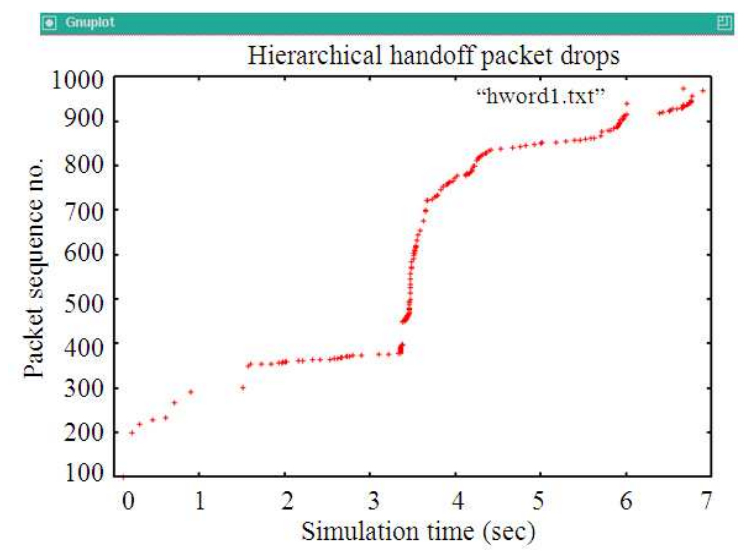

Fig. 6: Packet loss hierarchical handoff

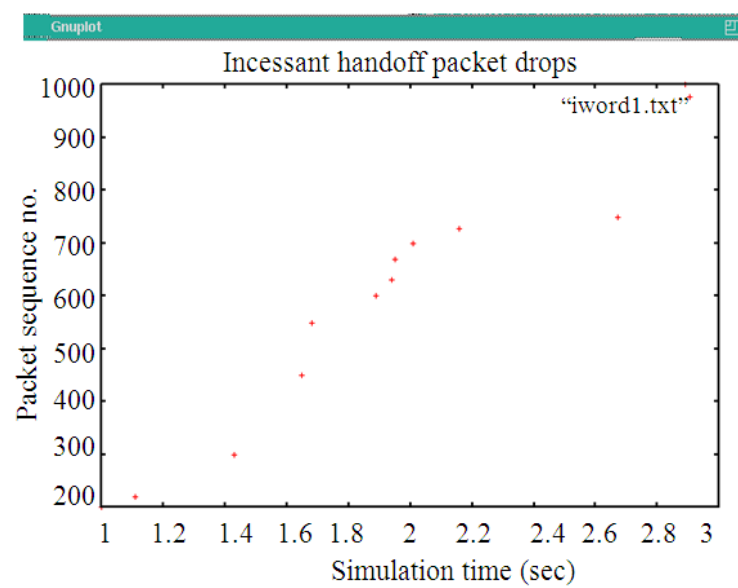

Fig. 7: Packet loss incessant hand off

\section{DISCUSSION}

Registration delay: The following Table 3 and graphs compares the delays of various handoff mechanisms like hierarchical intra domain, hierarchical inter domain, fast handoff, incessant inter domain and incessant intra-domain. Among the various mechanisms it is evident that incessant handoff mechanism takes very less delay time compared to all other existing mechanisms.

Packet loss: The following Table 4 and graphs compares the packet drops of various handoff mechanisms like hierarchical, fast handoff and incessant handoff mechanism. Among the various mechanisms it is evident that incessant handoff mechanism results in very less packet loss compared to all other existing mechanisms.

Table 3: Registration delay analysis

\begin{tabular}{lr}
\hline Handoff mechanism & Registration delay time $(\mathrm{m} \mathrm{sec})$ \\
\hline Fast Handoff & 6.25 \\
Hierarchical Intra-domain & 3.75 \\
Hierarchical Inter-domain & 6.75 \\
Incessant Intra-domain & 1.50 \\
Incessant Inter-domain & 2.50 \\
\hline
\end{tabular}

Table 4: Packet loss analysis

\begin{tabular}{lr}
\hline Handoff mechanism & Packet loss \\
\hline Fast Handoff Mechanism & 109 \\
Hierarchical Handoff Mechanism & 170 \\
Incessant Handoff Mechanism & 11 \\
\hline
\end{tabular}

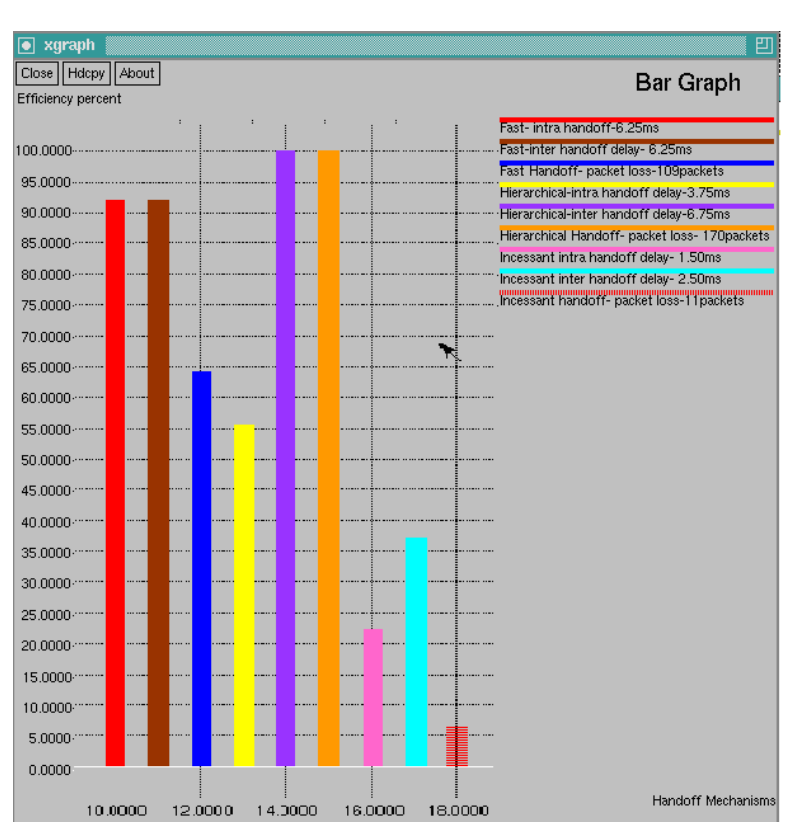

Fig. 8: Comparative analysis of existing and proposed mechanisms 
The following bar graph Fig. 8, compares the overall results of existing and proposed mechanisms. This graph clears that the incessant hand off mechanism is performing well in simulation environment.

\section{CONCLUSION}

There is a significant user demand over the reduction of handoff registration delays in ubiquitous wireless communication and the need for mounting network mobility in real time implementation. We present a precise analysis over sub-optimality of present handoff mechanisms and provide a solution to curb their drawbacks by projecting a new proposal for handoff mechanism. Based on the analysis of simulated scenario it has been proved that the incessant system Incessant Handoff Mechanism takes a lead over other mechanisms and provides higher efficiency and performance.

\section{REFERENCES}

Abdelgadir, A.T., M. Ahmed, A.S.K. Pathan, M.A. Abdullah and S. Haseeb, 2011. Performance analysis of a highly available home agent in mobile networks. Proceedings of the 14th Communications and Networking Symposium, Spring Simulation Multi-Conference, Apr. 03-07, IEEE Xploor Press, Boston, USA., pp: 62-70.

Beijnum, I.V., 2006. IPv6 Intervals. Goldstein Report. Bernardos, C.J., A.D.L. Olivia, M. Calderon, D.V. Hugo and H. Kahle, 2005. NEMO: Network Mobility. Bringing Ubiquity to the Internet Access. Universidad Carlos III de Madrid.

QS, 2011. University carlos III de madrid. Quacquarelli Symonds Limited.
Castelluccia, C., 1998. A Hierarchical Mobile IPv6 Proposal. Institut National De Recherche En Informatique Et En Automatique.

Devarapalli, V., R. Wakikava, A. Petrescu and P. Thubert, 2005. RFC 3963, Network Mobility Basic Support Protocol. Network Working Group.

Huang, P.J., Y.C. Tseng and K.C. Tsai, 2006. A fast handoff mechanism for IEEE 802.11 and IAPP networks. Proceedings of the IEEE 63rd Vehicular Technology Conference, May 7-10, IEEE Xploor Press, Melbourne, pp: 966-9701. DOI: 10.1109/VETECS.2006.1682968

Nasir, M.H.N.M., H. Hassan and N. Jomhari, 2008. The use of mobile phones by elderly: A study in malaysia perspectives. J. Soc. Sci., 4: 123-127. DOI: 10.3844 jssp.2008.123.127

Johnson, D.B. A.D. Joseph and N.H. Vaidya, 2003. A comparison of mechanisms for improving mobile IP handoff latency for end-to-end TCP. Proceedings of the 9th Annual International Conference on Mobile Computing and Networking, Sep. 14-19, ACM, New York, USA., pp: 29-41. DOI: 10.1145/938985.938989

Hassan, S.S. and R. Hassan, 2011, IPv6 Network Mobility Route Optimization Survey. Am. J. Applied Sci., 8: 579-583. DOI: 10.3844/ajassp.2011.579.583

Sharma, S., N. Zhu and T.C. Zhiueh, 2004. Lowlatency mobile IP handoff for infrastructure-mode wireless LANs. IEEE J. Selec. Areas Commun., 22: 643-652. DOI: 10.1109/JSAC.2004.825988

Sulander, M., T. Hamalainen, A. Viinikainen and J. Puttonen, 2005. A Fast Handover Method for RealTime Multimedia Services. IGI Global. DOI: 10.4018/978-1-59140-866-6.ch013 